Published in final edited form as:

Menopause. 2016 February ; 23(2): 150-157. doi:10.1097/GME.000000000000510.

\title{
Carotid Artery Distensibility and Hormone Therapy and Menopause: The Los Angeles Atherosclerosis Study (LAAS)
}

\author{
Chrisandra Shufelt, MD, MS ${ }^{1,{ }^{*}}$, Omeed Elboudwarej, MD $^{1,{ }^{\star}}$, B. Delia Johnson, $\mathrm{PhD}^{2}$, Puja \\ Mehta, MD ${ }^{1}$, Vera Bittner, MD, MSPH ${ }^{3}$, Glenn Braunstein, MD ${ }^{1}$, Sarah Berga, MD ${ }^{4}$, Frank \\ Stanczyk, $\mathbf{P h D}^{5}$, Kathleen Dwyer, $\mathbf{P h D}^{6}$, and C. Noel Bairey Merz, MD ${ }^{1}$ \\ ${ }^{1}$ Barbra Streisand Women's Heart Center, Cedars-Sinai Heart Institute, and Department of \\ Medicine, Cedars-Sinai Medical Center, Los Angeles, CA \\ 2University of Pittsburgh, Pittsburgh, PA \\ ${ }^{3}$ Division of Cardiovascular Disease, University of Alabama at Birmingham, Birmingham, AL \\ ${ }^{4}$ Department of Obstetrics and Gynecology, Wake Forest School of Medicine \\ ${ }^{5}$ Departments of Obstetrics and Gynecology and Preventive Medicine, Keck School of Medicine, \\ University of Southern California, Los Angeles, CA \\ ${ }^{6}$ Department of Preventive Medicine, Institute for Prevention Research, Keck School of Medicine, \\ University of Southern California, Los Angeles, CA
}

\section{Abstract \\ Objective-Observational studies suggest that arterial distensibility decreases during menopause; however, the relation to hormone therapy use is controversial. We prospectively studied distensibility and hormone therapy use during different menopause stages.}

Methods-161 women between 42-61 years of age without cardiovascular disease had carotid artery measurements by ultrasound to calculate the distensibility index at baseline and 3 years

\footnotetext{
Address for correspondence: Chrisandra Shufelt, MD, 8631 W. Third Street, Suite 740 East, Los Angeles, California 90048, Phone: (310) 423-9660, Fax: (310) 423-9668, shufeltc@cshs.org.

Both contributed equally

Disclaimers

None

Conflicts of Interest and Source of Funding

Chrisandra Shufelt: none

Omeed Elboudwarej: none

B. Delia Johnson: none

Puja Mehta: Gilead, General Electric

Vera Bittner: none

Glenn Braunstein: none

Sara Berga: Agile Therapeutics Medical Advisory Board; AHC Media, LLC - Consultant, Annual Business Meeting; Ironwood Pharmaceuticals; Noven Pharmaceutical Medical Advisory Board; Pfizer, International Menopause Society 2011 Conbriza Symposium; Pfizer, Scientific Advisory Board, Estrogens, SERMS, and TSECS; Shionogi, Inc, Consultant -Medical Advisory Board; Teva Pharmaceutical Industries, Ltd Expert Advisory Board Frank Stanczyk: none

Kathleen Dwyer: none

C. Noel Bairey Merz: Allegheny General Hospital; Bryn Mawr Hospital; Duke; Emory; FAMRI; Garden State AHA; Gilead; Japanese Circ Society; Kaiser Permanente; Mayo Foundation; NIH-SEP; PCNA; Practice Point Communications; Research Triangle Institute International; RWISE; UCSF; University of New Mexico; Victor Change Cardiac Research Institute; Vox Media; WISE CVD
} 
later. Menopause stage was classified at each visit as premenopausal, perimenopausal, and postmenopausal. Over 3 years of prospective observation, women were classified as remaining premenopausal, remaining postmenopausal, or transitioning, defined as change from premenopausal-to-perimenopausal, premenopausal-to-postmenopausal, perimenopausal-toperimenopausal, or perimenopausal-to-postmenopausal.

Results-Distensibility declined over time in all menopause stages ( $\mathrm{p}<0.0001)$. Compared to postmenopausal women, premenopausal and transitioning/no hormone therapy women had over twice the decline in distensibility index ( $\mathrm{p}=0.06$ and $\mathrm{p}=0.016$, respectively), whereas transitioning/ hormone therapy did not differ in distensibility decline ( $\mathrm{p}=0.28)$. In a multivariate model, change in systolic blood pressure $(\mathrm{p}<0.0001)$ and change in pulse pressure $(\mathrm{p}=0.004)$ were independent predictors of distensibility index change and served as effect modulators. In the adjusted model, women in the premenopausal and transitioning/no hormone therapy groups had a significantly faster distensibility index decline ( $\mathrm{p}=0.002$ and 0.001 , respectively) than postmenopausal women, while the transitioning/hormone therapy group $\operatorname{did}$ not $(\mathrm{p}=0.21)$

Conclusions-These findings confirm that menopause transition is associated with reduced vascular compliance. Hormone therapy is associated with better arterial distensibility only during menopause transition. Additional prospective studies are needed to confirm these findings and to determine if hormone therapy use beyond menopause transition is related to distensibility.

\section{Keywords}

arterial distensibility; menopause; hormone therapy; estrogen; progesterone

\section{Introduction}

Arterial distensibility has emerged as a useful prognostic indicator for cardiovascular disease (CVD) beyond standard risk factors, such as hypertension, hyperlipidemia, and diabetes. ${ }^{1}$ Distensibility is a measure of an artery's ability to expand in response to changes in pulse pressure and an inverse measure of arterial stiffness. Loss of distensibility has been associated with the development of CVD, hypertension, and stroke in healthy individuals. ${ }^{2-4}$ In addition, markers of arterial distensibility, such as pulse pressure, have helped clarify the results of trials showing different clinical outcomes with differing antihypertensive therapy regimens despite similar reductions in blood pressure. ${ }^{5}$

With aging, CVD risk factors shift unfavorably for women and coincide temporally with a decline in estrogen levels and menopause. Cross-sectional data examining the influence of menopause transition on arterial distensibility have primarily shown an acceleration of agerelated increase in stiffness, ${ }^{6-9}$ however, cross-sectional data cannot test independent relations between distensibility and age versus menopausal hormonal changes. ${ }^{10,11}$ Additionally, the impact of hormone therapy (HT) on arterial distensibility still remains controversial as some observational studies suggest a benefit while others have not found HT to have a substantive impact. ${ }^{12-14}$ Randomized controlled trials measuring distensibility in postmenopausal women on HT have have not shown a benefit to date, potentially because of later HT initiation after vascular changes have already occurred. ${ }^{15,16}$ 
The present study prospectively examines relations between menopause stage and changes in carotid artery distensibility over a 3-year period. It also examines the relationship of initiation of HT and distensibility. In addition, we sought to determine a relationship between arterial distensibility, hormonal change and CVD risk factors. We hypothesized that arterial distensibility would decline over time with concomitant reduction in estrogen levels, with the greatest decline occurring during menopause transition. Furthermore, we hypothesized that HT through estrogen replacement would be beneficially associated with these changes.

\section{Materials and Methods}

\section{Study Population}

The Los Angeles Atherosclerosis Study is a community-based cohort study of employees of a major utility company in southern California. All study subjects were recruited from the same company, from 4 different job categories: union, management, administrative, and other. Exclusion criteria were applied only at baseline and included a self-reported history of CVD (angina, myocardial infarction, revascularization, or stroke) and current treatment for cancer. Participants aged 40-60 years were randomly sampled from all employees within strata of sex, age, ethnicity, and smoking status. Hispanics and smokers were oversampled. The participation rate was $85 \%$ at study entry for a total cohort of 573 male and female participants, of which 280 were women. The population for the present analysis comprised 161 women aged 45-60 years at study entry who had sufficient information to determine menopausal status and distensibility index at baseline and 3-year follow up. The baseline examination was conducted between 1995 to 1996, and follow-up occurred 3 years later (mean SD: $3.0 \pm 0.24$ years). At baseline and 3-year visit, the women underwent evaluation of carotid intima-media thickness (cIMT). The study protocol was approved by the institutional review committee at Cedars-Sinai Medical Center and the University of Southern California, and all women provided written informed consent.

\section{Determination of menopause status}

We used the menopause status algorithm developed by the Women's Ischemia Syndrome Evaluation study to determine menopause stage at each study visit. This algorithm, which uses a combination of self-reported reproductive history and menstrual cycle pattern and dates with serum reproductive hormone levels, has been published elsewhere. ${ }^{17}$

Reproductive hormone levels were assessed by the Los Angeles Atherosclerosis Study reproductive hormone core laboratory using validated steroid and protein assay methods for total estradiol, bioavailable estradiol, estrone, progesterone, FSH, and LH. ${ }^{18-20}$ In addition to using the menopause status algorithm, the Los Angeles Atherosclerosis Study Hormone Committee led by reproductive endocrinologists and scientists (S.L.B., G.D.B., and F.S.) reviewed and adjudicated menopause stage at baseline and at 3 years based on all reproductive and relevant biometric information. Menopause stage at baseline was categorized as premenopausal (Pre), perimenopausal (Peri), and postmenopausal (Post). At the 3-year visit, study participants were categorized as remaining Pre, remaining Post, or transitioning (Tran), defined as a change from Pre-to-Peri, Pre-to-Post, Peri-to-Peri, or Perito-Post. 


\section{Measurement of risk factors}

Study participants completed a nurse-administered questionnaire at each visit to determine demographic variables. A study nurse performed physical examinations and obtained height and weight measurements in light clothing. The seated blood pressure was determined using a standard sphygmomanometer with two separate readings after a minimum 10-minute rest. Serum and plasma samples were obtained after an overnight fast of at least 8 hours. HT use and name of hormone preparation were also attained at baseline and follow-up visit.

\section{Distensibility Index}

The distensibility index (DI) was calculated using the following formula ${ }^{21}$ :

$$
(2 / \text { diastolic diameter of common carotid artery }[C C A]) \times([\text { diastolicsystolic }
$$
diameter of $C C A] /$ pulse pressure)

Diastolic and systolic internal diameter of the CCA was measured as part of the cIMT protocol previously published. ${ }^{22}$ While cIMT was measured at each of the four visits, the systolic and diastolic internal diameters needed to calculate the DI were only measured at baseline and at the 3-year follow-up visit.

\section{Statistical methods}

Continuous variables are summarized as means \pm standard deviation (SD) and dichotomous variables are reported as percentages. Highly skewed variables are reported as medians (interquartile range). Change in variables over time $(\Delta)$ was obtained by subtracting baseline values from those obtained at 3-year follow-up such that a positive value indicates an increase in the variable over time and a negative value a decrease. Differences across menopausal stages (Tables 1-3) were assessed by analysis of variance or non-parametric Kruskal-Wallis test, where appropriate.

All modeling used the generalized linear model (GLM) with menopausal status groups entered as a class variable. Differences in the mean baseline DI and mean change in DI among the menopausal status groups (Figure 1) used the CONTRASTS option. Prior to multivariate modeling, the distributions of all potential predictors were evaluated for deviations from normality and for clinically implausible outliers. Variables highly skewed to the right were log-transformed and those skewed to the left underwent power transformations. The DI was normally distributed and had no major outliers. To derive the final predictive model, all possible clinical and laboratory parameters from Table 1, with the exception of age, were first added, one at a time in separate models, to the menopausal stages in order to assess whether they were independent predictors of DI change and/or whether they affected the relationship in question. Variables with a $p<0.20$ or whose introduction in the model changed the relationship between menopausal stages and DI change were then entered into forward stepwise regression. This was followed by individually adding other possible covariates until the final model contained only significant covariates. Using the SOLUTION option in PROC GLM, the final model displays the pvalue across all menopausal groups as well as the parameters of each menopause stage compared to the reference group in addition to the independent covariates. Only 3 potential 
predictors had missing values (glucose, LDLc, and FSH), none of which entered the models. All tests were 2 -sided and $p \leq 0.05$ was considered statistically significant. All analyses were performed with SAS software version 9.3 (SAS Institute).

\section{Results}

The women's mean age was $51.5 \pm 4.6,85(53 \%)$ were white and $45(28 \%)$ Hispanic. The majority (90\%) were working in management or administrative positions, and there was no statistical difference between job position within each menopause group $(\mathrm{p}=0.38)$. The mean DI was $50.7 \pm 18.5$ at baseline and $38.9 \pm 12.8$ at 3 year follow-up. Among the 161 women, $17(11 \%)$ were Pre for the duration of the study and not using HT, including birth control pills. There were 52 women who were Tran (32\%), of whom 29 (56\%) were on HT (14 estrogen only, 1 progesterone only, 10 estrogen/progesterone, and 4 with unknown HT formulation). The remaining 92 women were Post (57\%) with 69 (75\%) of them on HT (38 estrogen only, 26 dual estrogen/progesterone, and 5 with unknown HT formulation).

The baseline characteristics for the study participants are shown in Table 1. As expected, there was a statistically significant difference in FSH as well as age between Pre, Tran, and Post ( $\mathrm{p}<0.0001)$. Additionally, use of anti-hypertensive medications and serum triglyceride levels were different with both anti-hypertensive use and triglycerides higher in the Tran/HT group ( $\mathrm{p}=0.047$ and $\mathrm{p}=0.037$, respectively). There were no significant differences among the remaining variables across the menopause stages.

The baseline hemodynamic variables across the menopause stages are shown in Table 2 . The mean baseline systolic blood pressure (SBP) was significantly different and lowest in Tran/No HT women ( $\mathrm{p}=0.024)$. Similarly, pulse pressure varied among all the groups $(\mathrm{p}=0.033)$. Among the variables used to calculate the baseline DI, the internal diameter of the CCA measured during diastole was relatively larger in Post women $(\mathrm{p}=0.037)$. The baseline DI was significantly different by menopause stage with the higher baseline DI in Pre and Tran/No HT as compared to Tran/HT and Post $(\mathrm{p}<0.0001)$.

The change over the 3-year period $(\Delta)$ in hemodynamic variables by menopause stage is shown in Table 3. With the exception of $\Delta \mathrm{FSH}$ and $\Delta \mathrm{DI}$ ( $\mathrm{p}<0.0001$ and $\mathrm{p}=0.046$, respectively), there were no statistical differences in other variables. The baseline DI and $\Delta \mathrm{DI}$ by menopause stage is illustrated in Figure 1 . The difference in $\Delta \mathrm{DI}$ between the Post women on HT versus not on HT was not statistically significant $(\mathrm{p}=0.38)$; therefore both subgroups were combined to form the reference group. Without covariates in the model, women in the Pre and Tran/No HT had over twice the decline in DI compared to Post women [beta (standard error) Pre: -7.09 (3.80), $\mathrm{p}=0.06$; Tran/No HT: -8.18 (3.36), $\mathrm{p}=0.016$ ] The DI decline in Tran/HT women did not differ statistically from that of the Post women $(-3.35$ [3.07], $\mathrm{p}=0.28)$.

We further evaluated change over time in risk factors as potential covariates. When adjusting for each covariate separately, two significant $(\mathrm{p}<0.0001)$ covariates (change in SBP and change in pulse pressure) enhanced the statistical relationship between menopause stage and DI ( $\mathrm{p}=0.019$ and 0.0003 , respectively). There was a strong correlation ( $\mathrm{r}=-0.71$ at 
baseline and $\mathrm{r}=-0.63$ at follow-up) between DI and pulse pressure. The only other significant covariate, change in heart rate, did not substantially modify the relationship between menopause stage and DI.

Table 4 presents the final multivariate model with Post used as the menopause stage reference and the independent covariates. The model $\mathrm{R}^{2}$ was 0.55 . Among the covariates, only change in heart rate $(\mathrm{p}=0.003)$ and change in pulse pressure $(\mathrm{p}<0.0001)$ remained independent predictors of change in DI. In comparison to the reference group Post, women in the Pre and Tran/No HT groups had a significantly faster DI decline ( $\mathrm{p}=0.002$ and 0.001 , respectively). In contrast, although the Tran/HT women did experience a somewhat faster DI decline compared to Post women, this difference was not statistically significant $(\mathrm{p}=0.21)$.

Because of strong collinearity with menopausal stage, age was not included in the final model. Adding baseline BMI ( $\mathrm{p}=0.51)$ or change in BMI $(\mathrm{p}=0.16)$, a potential confounder, to the model did not alter the significant relationship between DI decline and menopause status or the other covariates.

\section{Discussion}

Our prospective study demonstrates that DI significantly differs by menopause stage. At baseline, DI was higher in Pre and Tran/No HT women and lower DI in Tran/HT and Post women. Over a 3-year period, the largest decline in DI occurred in Tran/No HT group, while the lowest DI decline was found in the Tran/HT group as compared to Post. These findings suggest that prior to postmenopause, HT use during transition is beneficially associated with arterial distensibility decline. Furthermore, postmenopausal HT appears to have no beneficial relation to distensibility. Finally, our results suggest that a higher SBP and higher pulse pressure modifies the observation of menopause status and DI decline.

Our findings are consistent with a previous study of 93 premenopausal age-matched and 93 postmenopausal women not on HT. ${ }^{8}$ A significantly lower arterial distensibility was observed in postmenopausal women further suggesting that after controlling for age, estrogen status plays an important role in vascular function. Additionally, a lower distensibility was found to correlate with the number of years a woman was postmenopausal suggesting that distensibility is negatively associated with cumulative years of estrogen loss. Prior studies have also shown that menopause may augment the age-related increase in arterial stiffness. ${ }^{9,23}$ Staessen et al. evaluated distensibility and pulse wave velocity in 315 women as they transitioned through menopause over 5.2 years matched to $315 \mathrm{men}^{23}$ The findings were that arterial distensibility declined with age and after controlling for age, cardiovascular variables, and hormone use, Post women had a greater pulse wave velocity compared to Pre women. Additionally, these results could not be reproduced in the agematched male controls suggesting menopause and estrogen loss may contribute to arterial stiffness above the effects of age. Similarly, our study found the greatest decline in arterial distensibility occurred in Pre and Tran women not on HT and that this difference was statistically significant compared to Post women. 
Unlike most published studies examining arterial function and women on HT, our study is unique in that it further differentiates by menopause stages to assess the relationship between distensibilty and HT use. Our results indicate that because there was a slower decline in DI in Tran women on HT compared to Post, HT use during menopause transition may potentially slow the observed changes in arterial function. Prior studies show evidence that vascular changes may begin in the earliest stages of menopause and that menopause transition accelerates the age-related decline in endothelial function and stiffness. ${ }^{24,25} \mathrm{~A}$ number of studies have also shown that arterial distensibility declines more rapidly in women than men beginning around 45 years of age, coinciding with the stage of early menopause transition where estrogen fluctuations may begin. ${ }^{26,27}$ The change in distensibility is not related to gender difference such as arterial size or baseline compliance. ${ }^{22}$ This greater reduction in distensibility may contribute to the increased prevalence of systolic hypertension in older women and higher CVD risk. ${ }^{28}$

Observational studies investigating the impact of HT on vascular function in postmenopausal women have had mixed results, with some showing improvement in arterial elasticity ${ }^{12,13,29-32}$ and others indicating no benefit. ${ }^{14,21}$ Kawecka-Jaszcz et al. examined 76 postmenopausal women and found that there was significant improvement in pulse wave velocity, a surrogate for arterial compliance, after 3 months of HT. ${ }^{12}$ While not as robust, a retrospective study of 56 postmenopausal women also found that HT improved pulse wave velocity as early as 4 weeks. ${ }^{31}$ Interestingly, one study showed that after 1-month of withdrawing HT in 11 postmenopausal women resulted in significantly worse arterial compliance. ${ }^{13}$ To date however, randomized control trials have not demonstrated that HT in postmenopausal women results in vascular improvement. ${ }^{21,33}$ Additionally, recent results from the Kronos Early Estrogen Prevention study in early postmenopausal women did not find a difference in cIMT on HT, both transdermal and oral, compared to placebo. ${ }^{34}$ These data support our finding that HT use in Post women was not related to a significant change in DI decline compared to non-HT users.

While we did not observe a difference in change in DI when directly comparing Tran women on and Tran women off HT, our findings are consistent with a prior randomized trial of Tran women that found no difference in arterial distensibility or compliance at 6- and 24months after HT use compared to the placebo. ${ }^{16}$ It is possible that differences in arterial function between women on or off HT may not readily be apparent in Tran women since estrogen levels are still fluctuating. Other studies have shown that structural arterial changes, such as cIMT and adventitial diameter, may worsen as early as three months after the final menstrual cycle. ${ }^{24,35}$ Furthermore, while the effects of progesterone on vascular reactivity are not entirely clear, there is evidence to suggest that progesterone may counteract some of the vasodilatory effects of estrogen. ${ }^{36,37}$ Therefore, use of combination estrogen and progesterone HT in $34 \%$ of Tran women in our study may have attenuated the estrogenmediated changes in distensibility that would otherwise have been identified in the Tran period. Finally, it is possible that the spectrum of patients included as part of the Tran group, which included women from Pre-to-Peri to Pre-to-Post, was too broad to capture a significant change in vascular function over the 3 -year study period. 
In addition to aging, the decline in arterial distensibility during menopause transition may be related to lower serum estrogen levels. In addition to a direct atheroprotective effect, ${ }^{38}$ estrogen has been shown to reduce endothelial dysfunction and improve vascular reactivity over a short period of time. ${ }^{39-41}$ On a biochemical level, estrogen enhances endotheliumdependent vasodilatation via reduced endothelin release, stimulation of nitric oxide levels, and increased production of nitric oxide synthase, prostacyclin, and endothelium-derived hyperpolarizing factor. ${ }^{37}$ These estrogen-mediated effects may mitigate the effect of lowgrade inflammation, oxidative stress, and associated biomarkers of endothelial dysfunction that have been implicated in arterial stiffening. ${ }^{42,43}$

With respect to CVD risk factors, our study found that adding systolic blood pressure and pulse pressure to our final model affected the relationship between menopause and DI decline. Other studies have identified additional risk factors associated with reduced arterial distensibility, including obesity, ${ }^{44}$ daily insulin intake, ${ }^{45}$ smoking,${ }^{46}$ oxidized LDL,${ }^{42}$ African-American race, ${ }^{47}$ increased HDL,${ }^{47}$ and HIV.${ }^{48}$ While we assessed similar patient characteristics at baseline and follow-up, statistical significance was not reached. One reason why our study may not have found that these variables were statistically significant is that we assessed change in covariates over time as opposed to absolute values at baseline or follow-up.

Our results show that pulse pressure, which can serve as a marker of underlying arterial stiffness, increased across menopause stages. This has been demonstrated in a prior prospective study showing younger age at onset of menopause was associated with higher pulse pressure than women who underwent menopause at an older age. ${ }^{49}$ Higher pulse pressure has also been shown to be an independent predictor of adverse cardiovascular events. ${ }^{50}$ Post women with a higher baseline pulse pressure have more rapid progression in atherosclerosis. ${ }^{51}$ With regard to blood pressure, prior studies have shown a significant association between higher blood pressure and carotid stiffness. ${ }^{52,53}$ Similar to pulse pressure, arterial stiffness is an independent predictor of increased coronary events in patients with hypertension. ${ }^{54}$ The increase in arterial stiffness may increase blood pressure by creating a high pulse wave velocity that augments pressure. ${ }^{55}$ Advancing age in women is marked by predominance of sympathetic tone and decreased vagal tonic modulation of heart rate. ${ }^{56}$ Furthermore, there is a decrease in nitric oxide activity with aging and a diminished blood flow response to endothelium dependent and independent stimuli. ${ }^{57}$ There is evidence of hormonal involvement given the variation in radial artery distensibility seen during the menopause cycle. ${ }^{58}$ The rapid decline in distensibility during menopause transition may potentiate the increase in pulse pressure and SBP seen with aging, or vice versa.

\section{Potential study limitations}

Given the observational nature of this study, our findings indicate only an association between arterial distensibility and changes with menopause and HT use, not impact or causality. The transition through menopause is a gradual process that may not be accurately reflected by individual data points. However, the input of three reproductive endocrinologists in conjunction with the menopause status algorithm provides assurance 
about the classification of each study participant. In addition, the results of Table 2 provide construct validity demonstrating consistency with markers known to change over the menopause transition. It should be noted that while a comprehensive approach was taken to assess all hemodynamic variables and measures of DI, the results of the study could not be adjusted for unmeasured variables, such as physical activity. Therefore, we cannot definitely exclude that aging and use of HT may be surrogates for other factors not included in the study. Another potential limitation is that use of HT in this study was not randomized and that the conclusion that HT may impact arterial distensibility should be interpreted with caution. The variability in dose, type, and route of administration of HT could have led to differential effects on arterial distensibility. The generalizability of the results may also be limited by the fact that the participants in this study had no known CVD, and therefore the effects of HT on arterial distensibility during menopause transition in the setting of significant atherosclerosis cannot be deduced. Finally, the small number of participants in the menopause groups may have underpowered the study and masked other significant findings, such as other covariates that may be independent predictors of change in DI or a significant difference in DI decline comparing Tran and/or Post women on and off HT.

\section{Conclusion}

Pre and Tran women not using HT have the largest decline in arterial distensibility compared to Post women. These findings indicate that menopause transition is associated with reduced vascular compliance. HT appears to have an impact on arterial distensibility only if taken during the transitional period of menopause, indicating that the perimenopausal window presents a critical time during which changes to arterial function may occur that increase the risk of CVD. Additional prospective studies are needed to confirm these findings and to assess whether the impact of HT on arterial distensibility is sustained longterm.

\section{Acknowledgments}

This work was supported by contracts from the National Heart, Lung and Blood Institutes, nos. N01-HV-68161, N01-HV-68162, N01-HV-68163, N01-HV-68164, K23HL105787, HL-49910, HL-30568, grants U0164829, U01 HL649141, U01 HL649241, T32HL69751, R01-HL090957, 1R03AG032631 from the National Institute on Aging, GCRC grant MO1-RR00425 from the National Center for Research Resources and grants from the Gustavus and Louis Pfeiffer Research Foundation, Danville, NJ, The Women's Guild of Cedars-Sinai Medical Center, Los Angeles, CA, The Ladies Hospital Aid Society of Western Pennsylvania, Pittsburgh, PA, and QMED, Inc., Laurence Harbor, NJ, the Edythe L. Broad Women's Heart Research Fellowship, Cedars-Sinai Medical Center, Los Angeles, California, the Barbra Streisand Women's Cardiovascular Research and Education Program, Cedars-Sinai Medical Center, Los Angeles, The Society for Women's Health Research (SWHR), Washington, D.C., and the Linda Joy Pollin Women's Heart Health Program, Los Angeles, CA.

\section{References}

1. London GM, Cohn JN. Prognostic application of arterial stiffness: task forces. Am J Hypertens. Aug; 2002 15(8):754-758. [PubMed: 12160201]

2. Kaess BM, Rong J, Larson MG, et al. Aortic stiffness, blood pressure progression, and incident hypertension. Jama. Sep 5; 2012 308(9):875-881. [PubMed: 22948697]

3. Lebrun CE, van der Schouw YT, Bak AA, et al. Arterial stiffness in postmenopausal women: determinants of pulse wave velocity. J Hypertens. Nov; 2002 20(11):2165-2172. [PubMed: 12409954] 
4. Mattace-Raso FU, van der Cammen TJ, Hofman A, et al. Arterial stiffness and risk of coronary heart disease and stroke: the Rotterdam Study. Circulation. Feb 7; 2006 113(5):657-663. [PubMed: 16461838]

5. Williams B, Lacy PS, Thom SM, et al. Differential impact of blood pressure-lowering drugs on central aortic pressure and clinical outcomes: principal results of the Conduit Artery Function Evaluation (CAFE) study. Circulation. Mar 7; 2006 113(9):1213-1225. [PubMed: 16476843]

6. Izumi S, Muano T, Mori A, Kika G, Okuwaki S. Common carotid artery stiffness, cardiovascular function and lipid metabolism after menopause. Life Sci. Mar 6; 2006 78(15):1696-1701. [PubMed: 16266724]

7. Takahashi K, Miura S, Mori-Abe A, et al. Impact of menopause on the augmentation of arterial stiffness with aging. Gynecol Obstet Invest. 2005; 60(3):162-166. [PubMed: 15980658]

8. Westendorp IC, Bots ML, Grobbee DE, et al. Menopausal status and distensibility of the common carotid artery. Arterioscler Thromb Vasc Biol. Mar; 1999 19(3):713-717. [PubMed: 10073978]

9. Zaydun G, Tomiyama $\mathrm{H}$, Hashimoto $\mathrm{H}$, et al. Menopause is an independent factor augmenting the age-related increase in arterial stiffness in the early postmenopausal phase. Atherosclerosis. Jan; 2006 184(1):137-142. [PubMed: 15913634]

10. Casiglia E, Tikhonoff V, Boschetti G, et al. Arterial stiffness and related variables across menopausal status: an epidemiologic study. J Womens Health (Larchmt). Jan; 2013 22(1):75-84. [PubMed: 23305219]

11. O’Neill SM, Liu J, O'Rourke MF, Khoo SK. The menopausal transition does not appear to accelerate age-related increases in arterial stiffness. Climacteric. Feb; 2013 16(1):62-69. [PubMed: 23152960]

12. Kawecka-Jaszcz K, Czarnecka D, Olszanecka A, Rajzer M, Jankowski P. The effect of hormone replacement therapy on arterial blood pressure and vascular compliance in postmenopausal women with arterial hypertension. J Hum Hypertens. Jul; 2002 16(7):509-516. [PubMed: 12080436]

13. Rajkumar C, Kingwell BA, Cameron JD, et al. Hormonal therapy increases arterial compliance in postmenopausal women. J Am Coll Cardiol. Aug; 1997 30(2):350-356. [PubMed: 9247504]

14. Wong J, Wong S, Handa P, Abbott C. Hormone replacement use, arterial distensibility, cardiac structure and circadian blood pressure profile in menopausal women. Blood Press. 2005; 14(1):1220. [PubMed: 15823942]

15. Blacher J, Raison J, Amah G, Schiemann AL, Stimpel M, Safar ME. Increased arterial distensibility in postmenopausal hypertensive women with and without hormone replacement therapy after acute administration of the ACE inhibitor moexipril. Cardiovasc Drugs Ther. Sep; 1998 12(4):409-414. [PubMed: 9825188]

16. Westendorp IC, de Kleijn MJ, Bots ML, et al. The effect of hormone replacement therapy on arterial distensibility and compliance in perimenopausal women: a 2-year randomised trial. Atherosclerosis. Sep; 2000 152(1):149-157. [PubMed: 10996350]

17. Johnson BD, Merz CN, Braunstein GD, et al. Determination of menopausal status in women: the NHLBI-sponsored Women's Ischemia Syndrome Evaluation (WISE) Study. J Womens Health (Larchmt). Oct; 2004 13(8):872-887. [PubMed: 15671703]

18. Anderson DC, Hopper BR, Lasley BL, Yen SS. A simple method for the assay of eight steroids in small volumes of plasma. Steroids. Aug; 1976 28(2):179-196. [PubMed: 973234]

19. Rinaldi S, Geay A, Dechaud H, et al. Validity of free testosterone and free estradiol determinations in serum samples from postmenopausal women by theoretical calculations. Cancer Epidemiol Biomarkers Prev. Oct; 2002 11(10 Pt 1):1065-1071. [PubMed: 12376508]

20. Sodergard R, Backstrom T, Shanbhag V, Carstensen H. Calculation of free and bound fractions of testosterone and estradiol-17 beta to human plasma proteins at body temperature. J Steroid Biochem. Jun; 1982 16(6):801-810. [PubMed: 7202083]

21. Angerer P, Kothny W, Stork S, von Schacky C. Hormone replacement therapy and distensibility of carotid arteries in postmenopausal women: a randomized, controlled trial. J Am Coll Cardiol. Nov 15; 2000 36(6):1789-1796. [PubMed: 11092645]

22. Dwyer JH, Sun P, Kwong-Fu H, Dwyer KM, Selzer RH. Automated intima-media thickness: the Los Angeles Atherosclerosis Study. Ultrasound Med Biol. Sep; 1998 24(7):981-987. [PubMed: 9809632] 
23. Staessen JA, van der Heijden-Spek JJ, Safar ME, et al. Menopause and the characteristics of the large arteries in a population study. J Hum Hypertens. Aug; 2001 15(8):511-518. [PubMed: 11494087]

24. El Khoudary SR, Wildman RP, Matthews K, Thurston RC, Bromberger JT, Sutton-Tyrrell K. Progression rates of carotid intima-media thickness and adventitial diameter during the menopausal transition. Menopause. Jan; 2013 20(1):8-14. [PubMed: 22990755]

25. Moreau KL, Hildreth KL, Meditz AL, Deane KD, Kohrt WM. Endothelial function is impaired across the stages of the menopause transition in healthy women. J Clin Endocrinol Metab. Dec; 2012 97(12):4692-4700. [PubMed: 22969140]

26. Hansen F, Mangell P, Sonesson B, Lanne T. Diameter and compliance in the human common carotid artery--variations with age and sex. Ultrasound Med Biol. 1995; 21(1):1-9. [PubMed: 7754568]

27. Vermeersch SJ, Rietzschel ER, De Buyzere ML, et al. Age and gender related patterns in carotidfemoral PWV and carotid and femoral stiffness in a large healthy, middle-aged population. $\mathrm{J}$ Hypertens. Jul; 2008 26(7):1411-1419. [PubMed: 18551018]

28. Berry KL, Cameron JD, Dart AM, et al. Large-artery stiffness contributes to the greater prevalence of systolic hypertension in elderly women. J Am Geriatr Soc. Mar; 2004 52(3):368-373. [PubMed: 14962150]

29. Gorgulu S, Eren M, Celik S, et al. The effects of hormonal therapy on aortic stiffness and left ventricular diastolic function. Acta Cardiol. Feb; 2003 58(1):1-8. [PubMed: 12625488]

30. Liang YL, Teede H, Shiel LM, et al. Effects of oestrogen and progesterone on age-related changes in arteries of postmenopausal women. Clin Exp Pharmacol Physiol. Jun; 1997 24(6):457-459. [PubMed: 9171959]

31. Miura S, Tanaka E, Mori A, et al. Hormone replacement therapy improves arterial stiffness in normotensive postmenopausal women. Maturitas. Aug 20; 2003 45(4):293-298. [PubMed: 12927316]

32. McGrath BP, Liang YL, Teede H, Shiel LM, Cameron JD, Dart A. Age-related deterioration in arterial structure and function in postmenopausal women: impact of hormone replacement therapy. Arterioscler Thromb Vasc Biol. Jul; 1998 18(7):1149-1156. [PubMed: 9672076]

33. Hemelaar M, van der Mooren MJ, van Baal WM, Schalkwijk CG, Kenemans P, Stehouwer CD. Effects of transdermal and oral postmenopausal hormone therapy on vascular function: a randomized, placebo-controlled study in healthy postmenopausal women. Menopause. Sep-Oct; 2005 12(5):526-535. [PubMed: 16145306]

34. Harman S. Effects of Oral Conjugated Estrogen or Transdermal Estradiol Plus Oral Progesterone Treatment on Common Carotid Artery Initma Media Thickness (CIMT) and Coronary Artery Calcium (CAC) in Menopausal Women: Initial Results from the Kronos Early Estrogen Prevention Study (KEEPS). Menopause. Dec.2012 19(12):1365.

35. Wildman RP, Colvin AB, Powell LH, et al. Associations of endogenous sex hormones with the vasculature in menopausal women: the Study of Women's Health Across the Nation (SWAN). Menopause. May-Jun;2008 15(3):414-421. [PubMed: 18209686]

36. Luckas MJ, Gleeve T, Biljan MM, et al. The effect of progestagens on the carotid artery pulsatility index in postmenopausal women on oestrogen replacement therapy. Eur J Obstet Gynecol Reprod Biol. Feb; 1998 76(2):221-224. [PubMed: 9481579]

37. Orshal JM, Khalil RA. Gender, sex hormones, and vascular tone. Am J Physiol Regul Integr Comp Physiol. Feb; 2004 286(2):R233-249. [PubMed: 14707008]

38. Villablanca AC, Tenwolde A, Lee M, Huck M, Mumenthaler S, Rutledge JC. 17beta-estradiol prevents early-stage atherosclerosis in estrogen receptor-alpha deficient female mice. J Cardiovasc Transl Res. Sep; 2009 2(3):289-299. [PubMed: 19654889]

39. Ceylan-Isik AF, Erdogan-Tulmac OB, Ari N, Ozansoy G, Ren J. Effect of 17beta-oestradiol replacement on vascular responsiveness in ovariectomized diabetic rats. Clin Exp Pharmacol Physiol. Nov; 2009 36(11):e65-71. [PubMed: 19566816]

40. Higashi Y, Sanada M, Sasaki S, et al. Effect of estrogen replacement therapy on endothelial function in peripheral resistance arteries in normotensive and hypertensive postmenopausal women. Hypertension. Feb; 2001 37(2 Pt 2):651-657. [PubMed: 11230351] 
41. Miller VM, Mulvagh SL. Sex steroids and endothelial function: translating basic science to clinical practice. Trends Pharmacol Sci. Jun; 2007 28(6):263-270. [PubMed: 17466385]

42. Nawrot TS, Staessen JA, Holvoet P, et al. Telomere length and its associations with oxidized-LDL, carotid artery distensibility and smoking. Front Biosci (Elite Ed). 2010; 2:1164-1168. [PubMed: 20515788]

43. van Bussel BC, Schouten F, Henry RM, et al. Endothelial dysfunction and low-grade inflammation are associated with greater arterial stiffness over a 6-year period. Hypertension. Oct; 2011 58(4): 588-595. [PubMed: 21859964]

44. Moore XL, Michell D, Lee S, et al. Increased carotid intima-media thickness and reduced distensibility in human class III obesity: independent and differential influences of adiposity and blood pressure on the vasculature. PLoS One. 2013; 8(1):e53972. [PubMed: 23342053]

45. Cesana F, Giannattasio C, Nava S, et al. Impact of blood glucose variability on carotid artery intima media thickness and distensibility in type 1 diabetes mellitus. Blood Press. Dec; 2013 22(6):355-361. [PubMed: 23862738]

46. Ciftci O, Gunday M, Caliskan M, Gullu H, Guven A, Muderrisoglu H. Light cigarette smoking and vascular function. Acta Cardiol. Jun; 2013 68(3):255-261. [PubMed: 23882870]

47. Malayeri AA, Natori S, Bahrami H, et al. Relation of aortic wall thickness and distensibility to cardiovascular risk factors (from the Multi-Ethnic Study of Atherosclerosis [MESA]). Am J Cardiol. Aug 15; 2008 102(4):491-496. [PubMed: 18678312]

48. Zormpala A, Sipsas NV, Moyssakis I, et al. Impaired distensibility of ascending aorta in patients with HIV infection. BMC Infect Dis. 2012; 12:167. [PubMed: 22846182]

49. Luoto R, Sharrett AR, Eigenbrodt M, Arnett D. Pulse pressure and age at menopause. BMC Womens Health. Jun 28.2002 2(1):6. [PubMed: 12088509]

50. Safar ME. Systolic blood pressure, pulse pressure and arterial stiffness as cardiovascular risk factors. Curr Opin Nephrol Hypertens. Mar; 2001 10(2):257-261. [PubMed: 11224702]

51. Nair GV, Waters D, Rogers W, Kowalchuk GJ, Stuckey TD, Herrington DM. Pulse pressure and coronary atherosclerosis progression in postmenopausal women. Hypertension. Jan; 2005 45(1): 53-57. [PubMed: 15545509]

52. Giannarelli C, Bianchini E, Bruno RM, et al. Local carotid stiffness and intima-media thickness assessment by a novel ultrasound-based system in essential hypertension. Atherosclerosis. Aug; 2012 223(2):372-377. [PubMed: 22727194]

53. Beltran A, McVeigh G, Morgan D, et al. Arterial compliance abnormalities in isolated systolic hypertension. Am J Hypertens. Oct; 2001 14(10):1007-1011. [PubMed: 11710778]

54. Boutouyrie P, Tropeano AI, Asmar R, et al. Aortic stiffness is an independent predictor of primary coronary events in hypertensive patients: a longitudinal study. Hypertension. Jan; 2002 39(1):1015. [PubMed: 11799071]

55. Feihl F, Liaudet L, Waeber B. The macrocirculation and microcirculation of hypertension. Curr Hypertens Rep. Jun; 2009 11(3):182-189. [PubMed: 19442327]

56. Lavi S, Nevo O, Thaler I, et al. Effect of aging on the cardiovascular regulatory systems in healthy women. Am J Physiol Regul Integr Comp Physiol. Feb; 2007 292(2):R788-793. [PubMed: 16946083]

57. Al-Shaer MH, Choueiri NE, Correia ML, Sinkey CA, Barenz TA, Haynes WG. Effects of aging and atherosclerosis on endothelial and vascular smooth muscle function in humans. Int J Cardiol. May 10; 2006 109(2):201-206. [PubMed: 16054252]

58. Giannattasio C, Failla M, Grappiolo A, et al. Fluctuations of radial artery distensibility throughout the menstrual cycle. Arterioscler Thromb Vasc Biol. Aug; 1999 19(8):1925-1929. [PubMed: 10446072] 


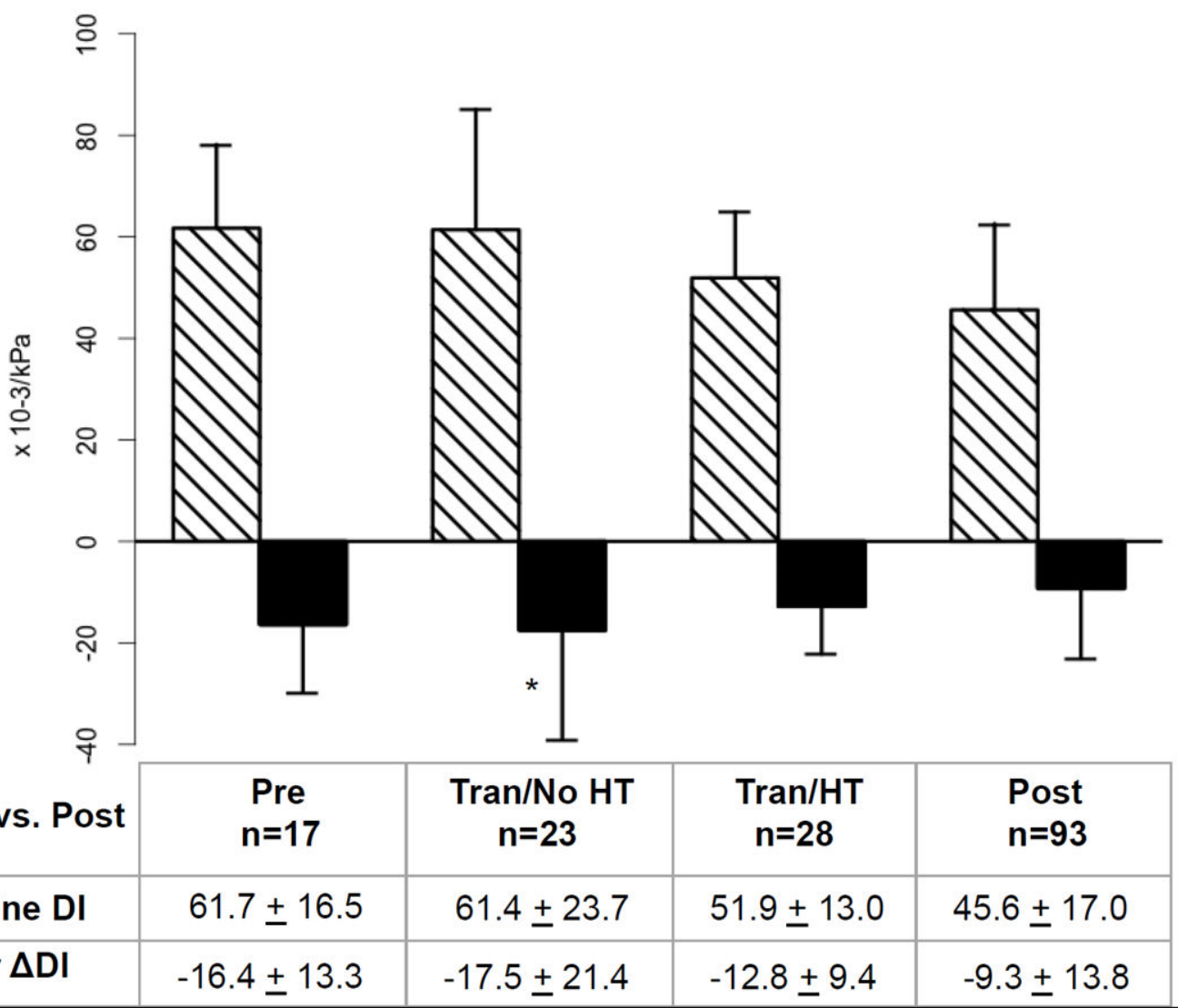

Figure 1. The baseline distensibility index (DI) and change in DI over 3-year period

There is a decline in DI over time as women transition through menopause. The rate of the decline is greatest in transitioning (Tran) women not taking hormone therapy (HT). The decline in DI is statistically significant $(\mathrm{p}=0.016)$ when comparing Tran/No HT women to postmenopausal women (Post). Abbreviations: HT, hormone therapy; Pre, premenopausal; Tran, transitioning; Post, postmenopausal. 


\section{Table 4}

Multivariate model of menopause stage and covariates on change in DI over 3-year period.

\begin{tabular}{|lcc|}
\hline Variable & Beta (standard error) & P-value \\
\hline Menopause group & $*$ & 0.0008 \\
Pre & $-8.2(2.6)$ & 0.002 \\
Tran/No HT & $-7.9(2.3)$ & 0.001 \\
Tran/HT & $-2.7(2.2)$ & 0.21 \\
Post & Reference & - \\
$\Delta$ Pulse Pressure & $-0.90(0.07)$ & $<0.0001$ \\
$\Delta$ Heart Rate & $-0.23(0.08)$ & 0.003 \\
\hline
\end{tabular}

Abbreviations: HT, hormone therapy; Pre, premenopausal; Tran, transitioning; Post; postmenopausal.

Since menopause group as a single variable is categorical, no beta (standard error) can be calculated 